\title{
Evolution of full phononic band gaps in periodic cellular structures
}

\author{
Maximilian Wormser $^{1}$ (D) Franziska Warmuth ${ }^{1} \cdot$ Carolin Körner $^{2}$
}

Received: 12 May 2017/ Accepted: 19 September 2017/Published online: 23 September 2017

(C) The Author(s) 2017. This article is an open access publication

\begin{abstract}
Cellular materials not only show interesting static properties, but can also be used to manipulate dynamic mechanical waves. In this contribution, the existence of phononic band gaps in periodic cellular structures is experimentally shown via sonic transmission experiment. Cellular structures with varying numbers of cells are excited by piezoceramic actuators and the transmitted waves are measured by piezoceramic sensors. The minimum number of cells necessary to form a clear band gap is determined. A rotation of the cells does not have an influence on the formation of the gap, indicating a complete phononic band gap. The experimental results are in good agreement with the numerically obtained dispersion relation.
\end{abstract}

\section{Introduction}

Metamaterials are artificially manufactured materials with counter-intuitive properties usually not found in nature [1]. Besides mechanical metamaterials (e.g., negative Poisson's ratio [2-4]) and optical metamaterials (e.g., negative index of refraction $[5,6]$, a general review on photonic metamaterials is given in [7]). There are metamaterials that exhibit novel properties regarding their interaction with acoustic waves. One subset of these metamaterials are

Maximilian Wormser

maximilian.wormser@fau.de

1 Joint Institute of Advanced Materials and Processes (ZMP), Dr.-Mack-Strasse 81, 90762 Fürth, Germany

2 Chair of Materials Science and Engineering for Metals (WTM), University of Erlangen-Nuremberg, Martensstrasse 5, 91058 Erlangen, Germany phononic band gap materials in which the propagation of phonons is prohibited in one or more frequency ranges [8]. The phononic band gap (PBG) is an intriguing property that offers many possible applications, e.g., sonic insulation [9-11], vibration control [12-14] or acoustic wave guides [10, 15, 16]. Since their initial discovery in 1992 [17] and their experimental proof some years later [18, 19], PBGs have attracted a lot of interest in the scientific community.

There are many examples for the numerical calculation of PBGs in periodic structures based on unit cells with periodic Bloch-Floquet boundary conditions [20-24]. In contrast, the number of publications showing experimental verification of phononic band gaps in three-dimensional structures is small. Typically, shakers and accelerometers are used as means of excitation and sensing of the acoustic waves in a sonic transmission experiment [11, 13, 25, 26]. These methods are not suited for high-frequency ultrasonic sound waves, though. Piezoceramic modules are a viable alternative for these experiments, since they work in the ultrasonic as well as in the audible range. Additionally, they can function as sensor and actuator [27]. Numerical results already showed the existence of PBGs for a specific eigenmode of a strut-based cubic unit cell [28, 29]. In contrast to phononic crystals which usually consist of two distinct, periodically arranged materials with a mismatch of density and elastic constants [1], the material presented in this paper consists of only one phase. In these materials, PBGs emerge from a sophisticated design of the cellular structure.

In the following, we present the experimental verification of the previously found numerically predicted PBGs in cellular structures. The existence of complete PBGs in the presented cellular structures manufactured from Ti-6Al$4 \mathrm{~V}$ powder by selective electron beam melting (SEBM) 
was already shown [30]. This study is to this date the first one to investigate experimentally how many cells are necessary to observe a PBG. To this end, samples with varying numbers of unit cells are examined in a sonic transmission experiment to identify the PBGs. Additionally, the influence of the orientation of the structures is investigated. Finally, the results are compared to a numerically determined dispersion relation.

\section{Methods}

\subsection{Sample preparation}

The samples consist of a specific unit cell that was arranged using a CAD (computer aided design) software (see Fig. 1a). This unit cell corresponds to the 61 st eigenmode of the cubic unit cell with periodic boundary conditions [29, 31]. The node distance (i.e., where the struts intersect) is $5 \mathrm{~mm}$, the amplitude of the struts is $1 \mathrm{~mm}$ and the strut thickness in the CAD file is $0.25 \mathrm{~mm}$, although the resulting strut thickness of the manufactured part is mainly governed by the SEBM parameters. All samples have a width and height of $5.5 \times 5.5$ cells with varying lengths of $1,2,3,4,5.5,8$, 10.5 and 13 unit cells. At two opposing sides, a thin wall covering the whole cross section is added to the structure to offer a flat surface for mounting the actuator and sensor by gluing. A finished sample and a close-up of the cellular structure can be seen in Fig. 1b, c.

Furthermore, samples with rotated unit cells $\left(15^{\circ}, 30^{\circ}\right.$, $45^{\circ}$; see Fig. 2) are built. Another sample with arbitrary rotation around all axes $\left(85^{\circ}, 32^{\circ}, 58^{\circ}\right.$ for $x, y$ and $z$, respectively) is constructed. The size of the rotated samples is identical to a $5.5 \times 5.5 \times 5.5$ cells sample.

The structures are built layer by layer in the SEBM process using an ARCAM AB Q10 machine. Details on the process can be found in other publications (e.g., [32]) and will not be further discussed here. The material used in the process is $\mathrm{Ti}-6 \mathrm{Al}-4 \mathrm{~V}$. The process parameters of the
SEBM process are identical for all samples used in this study (preheating temperature $T=730^{\circ} \mathrm{C}$, chamber pressure $p=7 \times 10^{-6} \mathrm{bar}$, beam current $I=3 \mathrm{~mA}$, voltage $U=60 \mathrm{kV}$, line speed $v=450 \mathrm{~mm} \mathrm{~s} \mathrm{~s}^{-1}$, line energy $E=0.4 \mathrm{~J} \mathrm{~mm}^{-1}$ ).

On the flat surfaces (approx. $700 \mu \mathrm{m}$ thick) on the two opposing sides of the samples one $20 \mathrm{~mm} \times 30 \mathrm{~mm}$ piezoceramic modules (PI Ceramic) per side is applied using a thin layer of an acrylate-based glue. The two electrodes per piezoceramic module are contacted by soldering copper wire onto them.

The strut thickness was measured by computed tomography (CT) using a SCANCo MicroCT 40 for a single unit cell that was manufactured with the same parameters as the regular samples, although in a different run. It was calibrated using a $490 \mu \mathrm{m}$ titanium wire. The measurement with a threshold of 750 was done at $50 \mathrm{kV}$ voltage, $160 \mu \mathrm{A}$ current and $300 \mathrm{~ms}$ exposure time with a voxel size of $15 \mu \mathrm{m}$ and an image size of $1024 \times 1024$ pixels.

\subsection{Impedance and sonic transmission measurement setup}

To gain a better knowledge of possible influences of the piezoceramic module on the transmission results, we measure its impedance in the same frequency range where the sonic transmission is recorded. The impedance analysis is conducted on an HP IMPEDANCE ANALYzer 4194 A. The impedance is measured from one electrode of a piezoceramic module already applied to a sample to its other electrode.

The principle of the sonic transmission measurement setup (see Fig. 3) is to measure an incoming signal and compare it to the outgoing signal to see how much the transmitted signal is attenuated by the structure. Creating and measuring the acoustic wave is done by the piezoceramic actuator and sensor, respectively. The actuator is connected to the function generator (HAMEG HMF-2525) which applies a sine wave function with a $10 \mathrm{~V}$ amplitude.
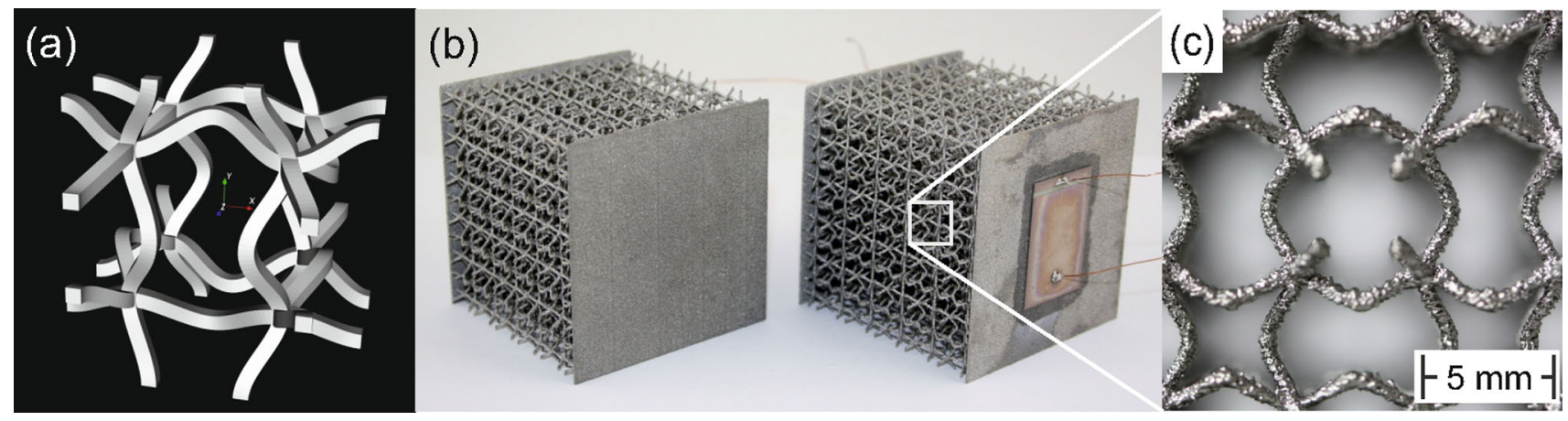

Fig. 1 a CAD view of the unit cell which is the 61st eigenmode of a cubic cell with periodic boundary conditions. b Test samples made from Ti$6 \mathrm{Al}-4 \mathrm{~V}$ by SEBM with applied piezoceramic actuator and sensor on the right sample. c Close-up view of the cellular structure 


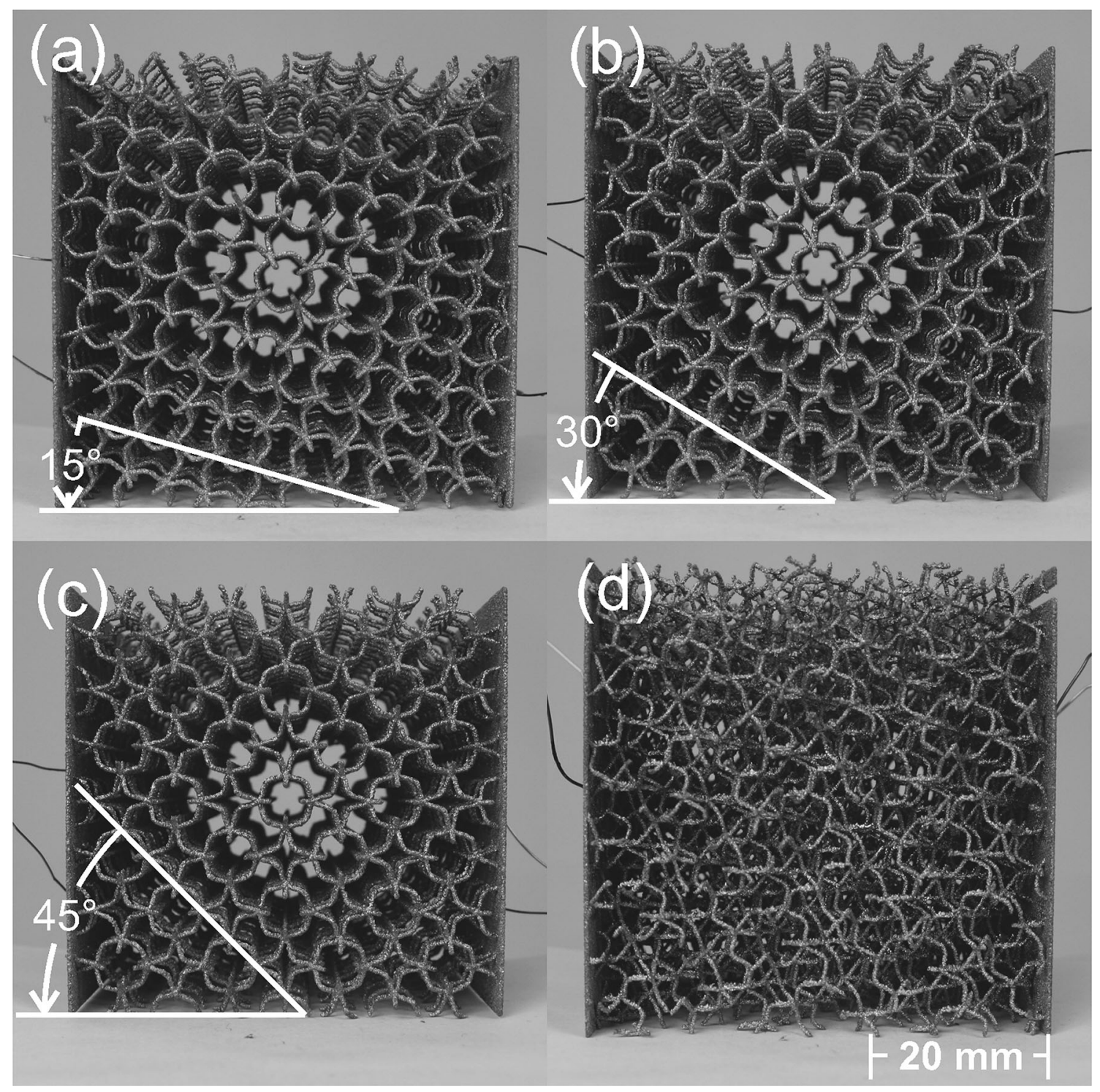

Fig. 2 Photographs of samples with $\mathbf{a} 15^{\circ}, \mathbf{b} 30^{\circ}$ and $\mathbf{c} 45^{\circ}$ rotation around the length-axis of the sample. $\mathbf{d}$ A sample with rotation around all three axes

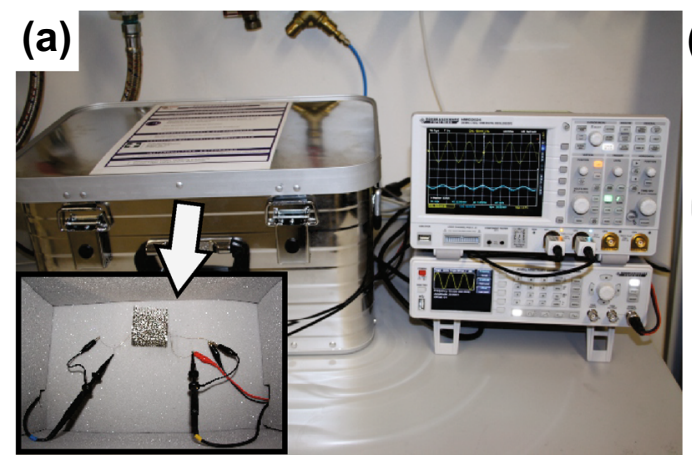

Fig. 3 a The sonic transmission setup with the oscilloscope and the function generator on the top right and bottom right, respectively. The inset shows how the samples are connected to the probes from the

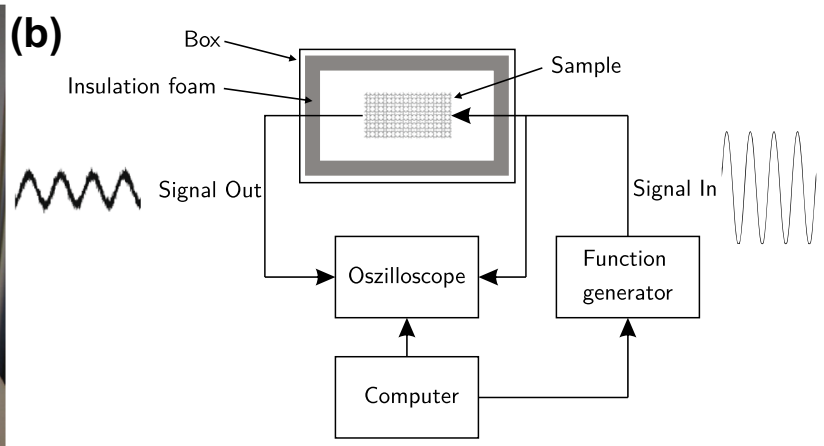

oscilloscope inside the of the box. b A schematic of the functional principle of the setup 
The oscilloscope (HAMEG HMO-2024) probes the signal at the actuator and the signal at the sensor on the opposing side of the sample. This measurement is conducted in steps of $1 \mathrm{kHz}$, where each frequency is measured 64 times and automatically averaged by the oscilloscope. The computer automatically increments the frequency and saves the data using an in-house developed program. The sample is situated inside an aluminum box that is lined with insulating foam to prevent external influences from impacting the measurement.

\subsection{Numerical calculation of the dispersion relation}

In order to numerically identify the PBGs of a periodic cellular structure, a dispersion relation has to be calculated. We determine the dispersion relation of the unit cell of Fig. 1 using COMSOL Multiphysics 5.1. Periodic BlochFloquet boundary conditions are applied. A more detailed description of the method can be found in a previous publication [29].

\section{Results and discussion}

\subsection{Impedance of the piezoceramic sensor/actuator module}

The impedance analysis of the piezoceramic modules mounted on the samples is depicted in Fig. 4. Obviously, the piezoceramic actuators do not have a steady response behaviour. They show local maxima and minima at their (anti-)resonance frequencies which are located at around $50^{\circ}, 75^{\circ}, 125 \mathrm{kHz}$. A sonic transmission spectrum is plotted alongside the impedance curve to be able to qualitatively compare their shapes. The local minima in the impedance analysis coincide with the peaks of the sonic transmission spectrum. Conversely, the transmission gets lower where the impedance rises. All the other actuators show the same behaviour with the same characteristic resonance frequencies. In consequence, the elongation of the piezoceramic is stronger (weaker) at these (anti-)resonance frequencies.

\subsection{Sonic transmission results for varying sample lengths}

Transmission is characterized by the ratio of outgoing to incoming signal amplitude. This ratio is plotted over the frequency for sample lengths from 1 to 13 cells in Fig. 5. The lowest value is up to four orders of magnitude lower than the highest value, indicating the occurrence of strong signal attenuation or loss. The regions of interest, i.e., the phononic band gaps, are frequency ranges with consistently

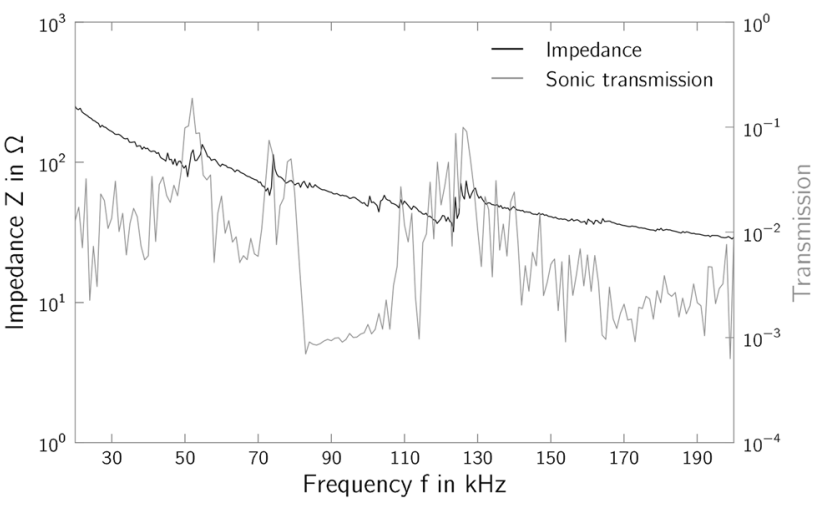

Fig. 4 A sonic transmission spectrum of the $5.5 \times 5.5 \times 5.5$ sample (grey) and an impedance analysis of an actuator applied to the sample (black)

low transmission ratios. A precise definition of experimentally determined PBGs is difficult, because criteria for the bounds of the PBG have to be chosen arbitrarily by lack of convention. Therefore, we will estimate the band gap position by eye where a clear and constant drop in transmission is visible.

While there is no PBG visible in the short samples, a PBG clearly forms with longer samples. At a length of 4 cells and higher the PBG has a uniform minimum with low variation as opposed to the noisy signal on either side. The position of the PBG is roughly the same for all samples beginning at $75-80 \mathrm{kHz}$ and ending at $100-110 \mathrm{kHz}$. The signal within the PBG is around one to two orders of magnitude smaller than the surrounding signals, usually at a transmission ratio of 0.001 . This also seems to be close to the resolution limit imposed by the oscilloscope.

For 8 cells or more, a weak second gap evolves between 160 and $180 \mathrm{kHz}$. While the gap is less pronounced here, the shape of the spectrum clearly indicates a region of interest. Especially the samples with a length of 10.5 and 13 cells show a smooth second PBG, comparable to the first PBG with at least 4 cells. The flat shape within the PBG is also observed in measurements with higher a resolution of $0.1 \mathrm{kHz}$ instead of $1 \mathrm{kHz}$. These finer measurements of the non-PBG parts of the spectrum look even noisier while the PBG regions remain smooth.

A comparable sample in an earlier publication [30] with a length of 8 cells ${ }^{1}$ showed a very clear second PBG, in contrast to the samples in this study. No definite reason for the lack of a second PBG in samples shorter than 10.5 cells can be given. A possible cause for the discrepancy can be a difference in application of the piezoceramic sensor/actor, e.g., a thinner or more uniform layer of glue.

\footnotetext{
${ }^{1}$ All parameters were the same for the earlier sample, except a higher line energy and lower line speed were used, resulting in thicker struts.
} 


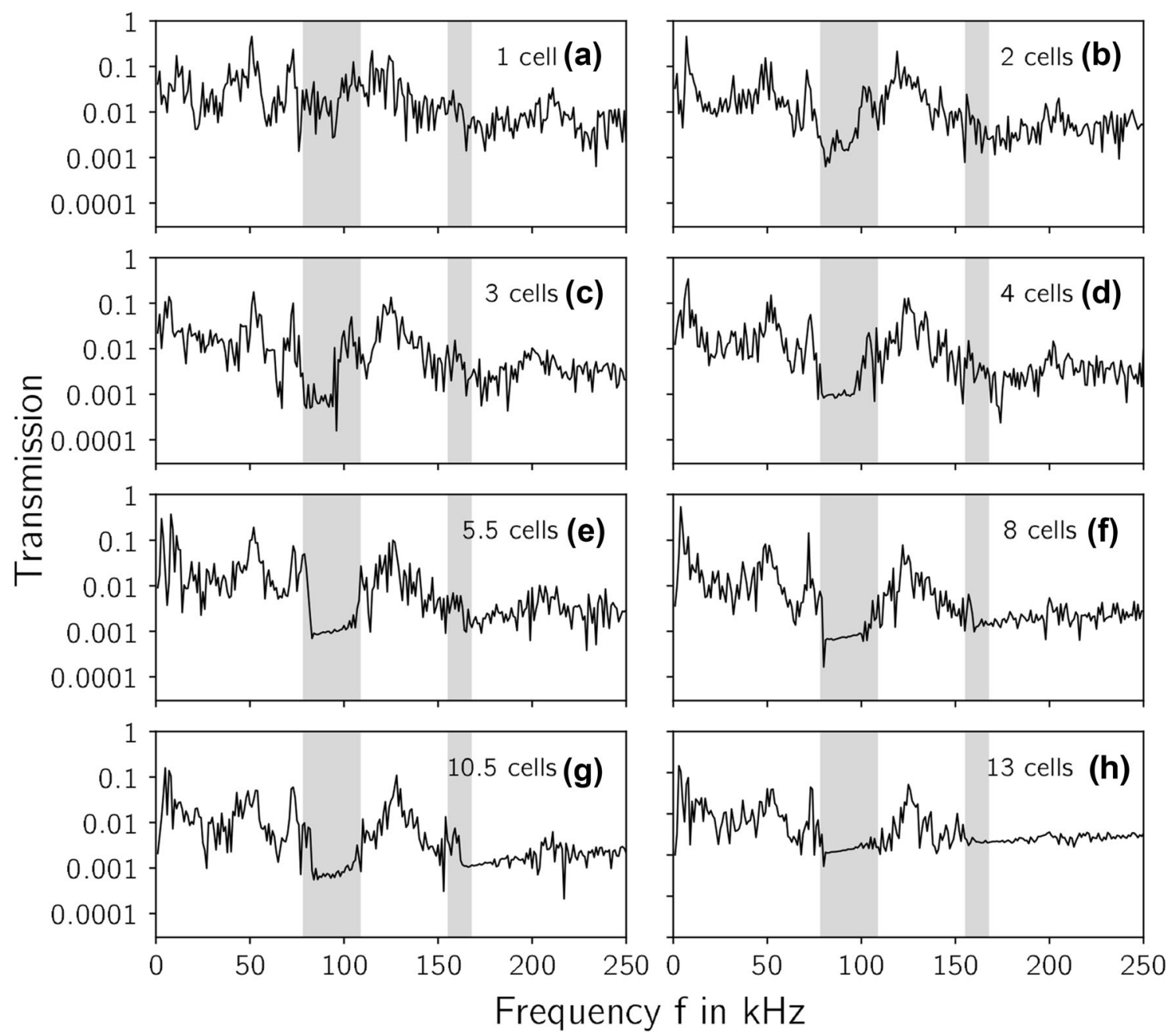

Fig. 5 Sonic transmission spectra for sample lengths of $\mathbf{a}-\mathbf{h}$ 1, 2, 3, 4, 5.5, 8, 10.5 and 13 cells. The grey areas indicate the PBGs calculated from a dispersion relation for a 0.45 -mm-thick strut

From these results we can deduce that about 4 cells in transmission direction are necessary for a well-defined PBG to emerge. More than 4 cells up to 13 do not appear to be detrimental to the depth or width of the PBG. The second PBG becomes visible at a sample length of at least 10.5 cells. The requirements for a clear second PBG will be subject to further investigations in the future.

In order to be able to compare the experimental data with numerical results, the thickness of the real samples has to be determined with a CT measurement of a unit cell. The CT measurement of the fabricated unit cell shows an average strut thickness of $0.4905 \pm 0.1165 \mu \mathrm{m}$. Given the large surface roughness and the fact that the roughness does not effectively contribute to the diameter of the strut in a mechanically relevant way [33], the dispersion relation was calculated for a slightly thinner strut thickness of $450 \mu \mathrm{m}$. The calculated PBGs from the dispersion relation are shown as grey bars in Fig. 5. For the first PBG they show good agreement with the experimental data. The second PBG is slightly lower and less wide in the numerical dispersion relation compared to the samples that show a second PBG. The dispersion relation is shown in detail in subsection 3.4.

\subsection{Sonic transmission results for samples with varying orientation with respect to the active axis}

The spectra for the rotated structures (see Fig. 6) qualitatively show the same results as the other samples. The PBG is visible and lies in the similar range between 70 and $100 \mathrm{kHz}$. The small deviation from the other samples can be explained by the fact that the sample series have been manufactured in different runs, possibly resulting in small differences in strut thickness. The sample rotated around all three axes shows the same behaviour as the other samples. This indicates a complete band gap, i.e., there is a PBG in all propagation directions of the mechanical wave. This 


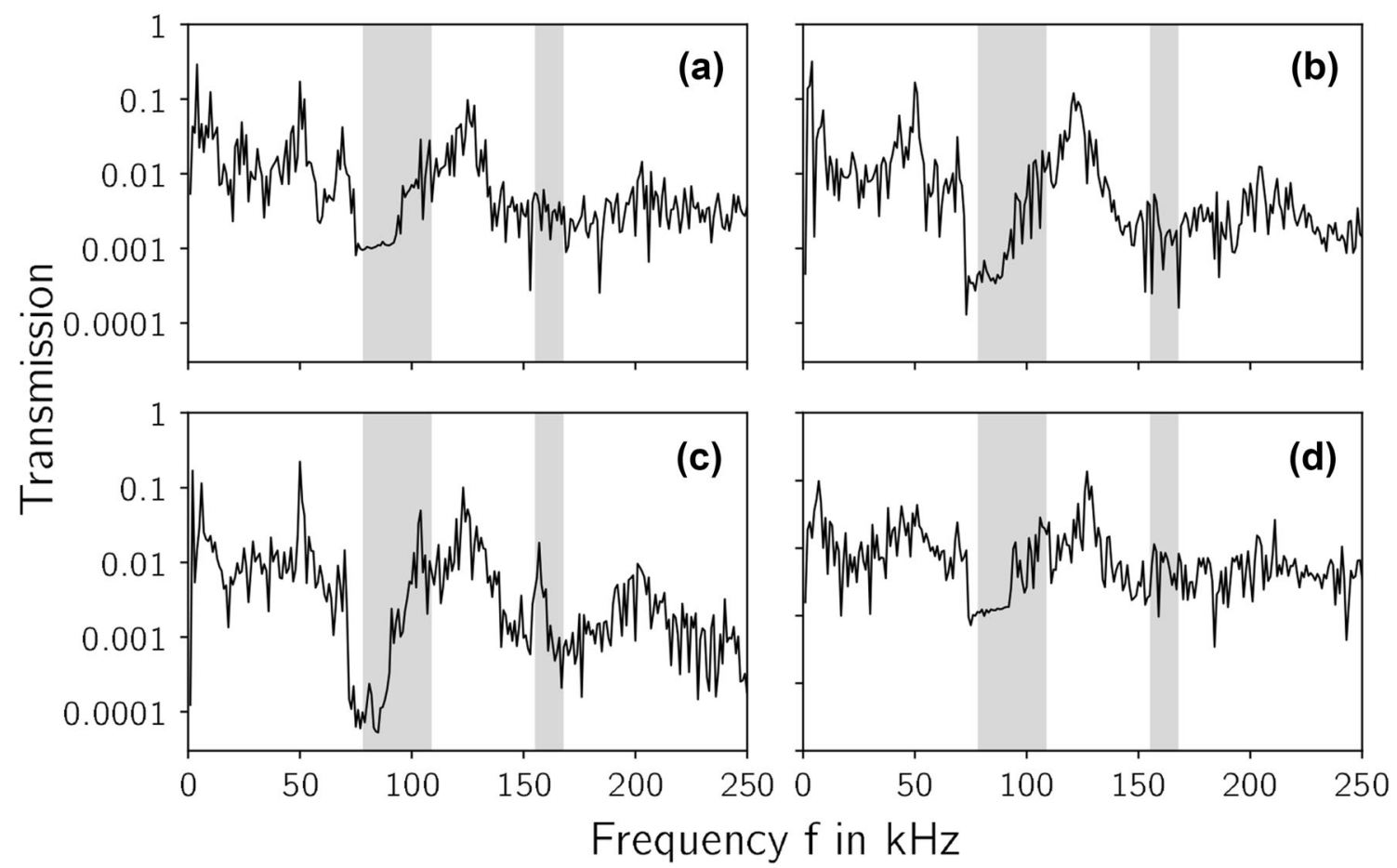

Fig. 6 Sonic transmission spectra for samples with rotations of $\mathbf{a} 15^{\circ}, \mathbf{b} 30^{\circ}$ and $\mathbf{c} 45^{\circ}$ of the sample and $\mathbf{d}$ an arbitrary rotation around all three axes. The grey areas indicate the PBGs calculated from a dispersion relation for a 0.45 -mm-thick strut

(b)

(a)

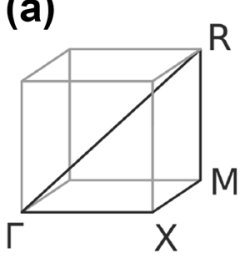

$\stackrel{\mathrm{k}_{\mathrm{z}}}{\mathrm{k}_{\mathrm{y}}}$

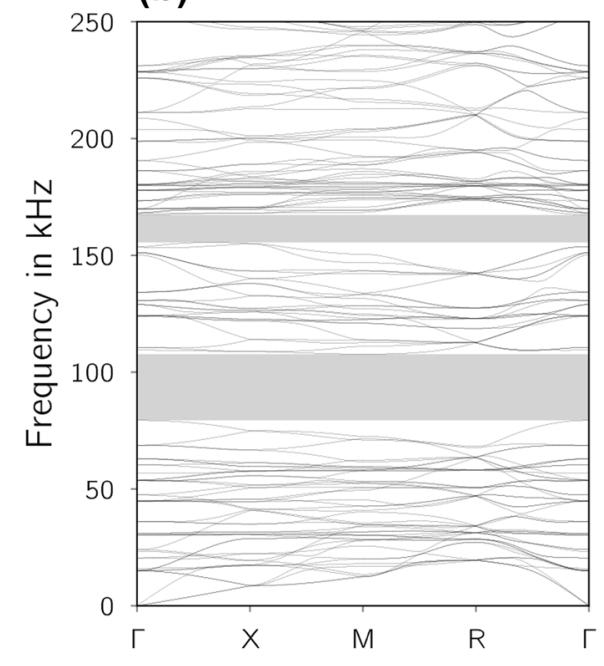

(c)

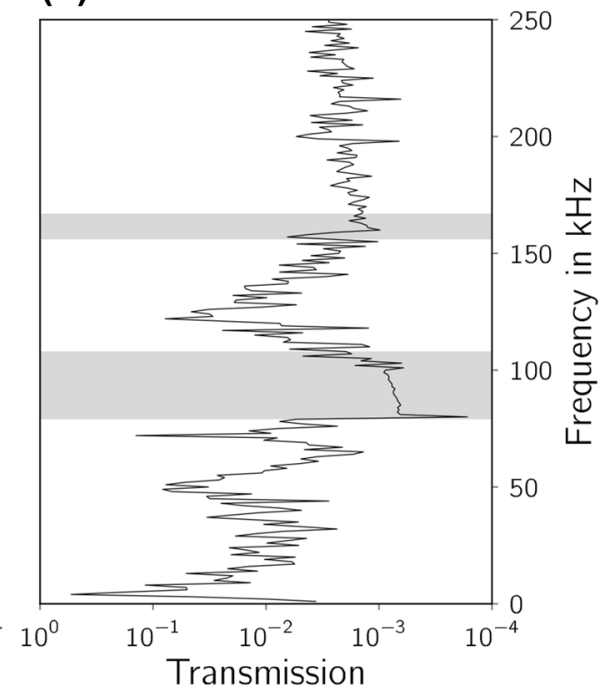

Fig. 7 a Unit cell of the reciprocal lattice. b Numerical dispersion relation of a unit cell with a strut thickness of $450 \mu \mathrm{m}$ along the reciprocal lattice points $\Gamma-\mathrm{X}-\mathrm{M}-\mathrm{R}-\Gamma$. $\mathbf{c}$ Transmission diagram for the

finding supports the results from a previous publication that already proved the existence of the PBG in the crystal directions (100), (110) and (111) [30].

There is no second PBG visible in any of the rotated samples. This is in line with the results of the last section, where a second PBG only emerged at a length of at least sample with dimensions $5.5 \times 5.5 \times 8$ cells. Complete PBGs obtained from the dispersion relation are marked in grey for both plots

10.5 cells, i.e., almost twice the length of the rotated samples. As with the samples in the last section, the resonance frequencies of the piezoceramic actuator do not interfere with the PBGs. The numerical results, again indicated by the grey bars, show good agreement with the experimental results for the first PBG. 


\subsection{Comparison of experimental and numerical results}

The numerical dispersion relation of a unit cell with $450 \mu \mathrm{m}$ strut thickness is shown in Fig. 7b. It shows two PBGs in the ranges from 79 to $108 \mathrm{kHz}$ and 156 to $167 \mathrm{kHz}$. The transmission diagram for the sample with dimensions $5.5 \times 5.5 \times 8$ cells (see Fig. $7 \mathrm{c}$ ) is shown in comparison and representatively for the other samples. The numerical result is in good agreement with the experimentally determined first PBG of the samples, as the alignment of the numerically determined PBG in grey with the transmission drop indicates. The second PBG, however, is not pronounced in the experimental data. This has been discussed in subsection 3.2. Since all samples show reasonably similar experimental PBGs, we can say that simulation and experiment are in good agreement for samples with varying length and orientation.

\section{Conclusion}

In this study, we examined the sonic transmission properties of a periodic cellular Ti-6Al-4V structure built by additive manufacturing (SEBM). The experiment showed that about 4 unit cells in transmission direction are necessary for a pronounced phononic band gap to evolve.

The PBG is still present if the number of cells increases or the structure is arbitrarily rotated. Longer samples with 10.5 cells or more showed a second phononic band gap. The lack of influence of the orientation of the sample is furthermore evidence for a complete PBG, as suggested by the numerical results. The experimentally determined positions and widths of the PBG are in good agreement with numerical results.

The reason for the weak second PBG needs to be investigated. Further research will focus on the effects of different geometric parameters to achieve wider and lower frequency PBGs with the underlying unit cell. A combination of different unit cells might open up possibilities to superpose PBGs.

Acknowledgements We gratefully acknowledge funding by the German Research Council (DFG) which, within the framework of its "Excellence Initiative", supports the Cluster of Excellence "Engineering of Advanced Materials" at the FAU Erlangen-Nürnberg. Furthermore, we would like to thank the Bavarian state for the funding of the Application Center VerTec. We also thank Manuel WeiSS and Stefan Rupitsch of the Chair of Sensor Technology of the FAU Erlangen-Nürnberg for fruitful discussions on the measurement setup and for letting us use the impedance analyzer.

Open Access This article is distributed under the terms of the Creative Commons Attribution 4.0 International License (http:// creativecommons.org/licenses/by/4.0/), which permits unrestricted use, distribution, and reproduction in any medium, provided you give appropriate credit to the original author(s) and the source, provide a link to the Creative Commons license, and indicate if changes were made.

\section{References}

1. M.H. Lu, L. Feng, Y.F. Chen, Mater. Today 12(12), 34 (2009). doi:10.1016/S1369-7021(09)70315-3

2. J. Schwerdtfeger, F. Schury, M. Stingl, F. Wein, R.F. Singer, C. Körner, Phys. Status Solidi B 249(7), 1347 (2011). doi:10.1002/ pssb.201084211

3. F. Warmuth, F. Osmanlic, L. Adler, M.A. Lodes, C. Körner, Smart Mater. Struct. 26(2), 025013 (2016). doi:10.1088/1361$665 \mathrm{X} / 26 / 2 / 025013$

4. N. Novak, M. Vesenjak, Z. Ren, Stroj. Vestn.-J. Mech. E. 62(9), 485 (2016). doi: $10.5545 /$ sv-jme. 2016.3656

5. H. Chen, C.T. Chan, P. Sheng, Nat. Mater. 9(5), 387 (2010). doi: $10.1038 /$ nmat 2743

6. R.A. Shelby, D.R. Smith, S. Schultz, Science 292(5514), 77 (2001). doi:10.1126/science.1058847

7. C.M. Soukoulis, M. Wegener, Nature Photonics (2011). doi:10. 1038/nphoton.2011.154

8. M.S. Kushwaha, P. Halevi, G. Martínez, L. Dobrzynski, B. Djafari-Rouhani, Phys. Rev. B 49(4), 2313 (1994). doi:10.1103/ PhysRevB.49.2313

9. Y. Pennec, J.O. Vasseur, B. Djafari-Rouhani, L. Dobrzyński, P.A. Deymier, Surf. Sci. Rep. 65(8), 229 (2010). doi:10.1016/j.surfrep. 2010.08.002

10. R.H. Olsson III, I. El-Kady, Meas. Sci. Technol. 20(1), 012002 (2009). doi:10.1088/0957-0233/20/1/012002

11. J. Wen, G. Wang, D. Yu, H. Zhao, Y. Liu, J. Appl. Phys. 97(11), 114907 (2005). doi:10.1063/1.1922068

12. F. Javid, P. Wang, A. Shanian, K. Bertoldi, Adv. Mater. (2016). doi:10.1002/adma.201600052

13. Y. Yan, Z. Cheng, F. Menq, Y.L. Mo, Y. Tang, Z. Shi, Smart Mater. Struct. 24(7), 075006 (2015). doi:10.1063/1.4891837

14. Y. Chen, T. Li, F. Scarpa, L. Wang, Phys. Rev. Appl. 7(2) (2017). doi: 10.1103/PhysRevApplied.7.024012

15. T. Miyashita, Meas. Sci. Technol. 16(5), R47 (2005). doi:10. 1088/0957-0233/16/5/R01

16. J.H. Sun, T.T. Wu, Phys. Rev. B 76(10) (2007). doi: 10.1103/ PhysRevB.76.104304

17. M.M. Sigalas, E.N. Economou, J. Sound Vib. 158(2), 377 (1992). doi:10.1016/0022-460X(92)90059-7

18. R. Martínez-Sala, J. Sancho, J.V. Sánchez, V. Gómez, J. Llinares, F. Meseguer, Nature 378(6554), 241 (1995). doi:10.1038/ $378241 \mathrm{a} 0$

19. J.V. Sánchez-Pérez, D. Caballero, R. Mártinez-Sala, C. Rubio, J. Sánchez-Dehesa, F. Meseguer, J. Llinares, F. Gálvez, Physical Review Letters 80(24), 5325 (1998). doi:10.1103/physrevlett.80. 5325

20. G. Trainiti, J. Rimoli, M. Ruzzene, Int. J. Solids Struct. 97-98, 431 (2016). doi:10.1016/j.ijsolstr.2016.07.006

21. A.S. Phani, J. Woodhouse, N.A. Fleck, J. Acoust. Soc. Am. 119(4), 1995 (2006). doi:10.1121/1.2179748

22. Y. Chen, H. Yao, L. Wang, J. Appl. Phys. 114(4), 043521 (2013). doi: $10.1063 / 1.4817168$

23. F. Lucklum, M.J. Vellekoop, I.E.E.E.T. Ultrason, Ferr. 63(5), 796 (2016). doi:10.1109/TUFFC.2016.2543527

24. P.A. Deymier (ed.), Acoustic Metamaterials and Phononic Crystals, 1st edn. (Springer Berlin Heidelberg, 2013). doi: 10. 1007/978-3-642-31232-8 
25. S. Shan, S.H. Kang, P. Wang, C. Qu, S. Shian, E.R. Chen, K. Bertoldi, Adv. Funct. Mat. 24(31), 4935 (2014). doi:10.1002/ adfm. 201400665

26. G. Trainiti, J.J. Rimoli, M. Ruzzene, In. J. Solids Struct. 75-76, 260 (2015). doi:10.1016/j.ijsolstr.2015.08.019

27. G. Park, C.R. Farrar, A.C. Rutherford, A.N. Robertson, J. Vib. Acoust. 128(4), 469 (2006). doi:10.1115/1.2202157

28. Y. Liebold-Ribeiro, C. Körner, Adv. Eng. Mater. 16(3), 328 (2014). doi:10.1002/adem.201300064

29. F. Warmuth, C. Körner, Materials 8(12), 8327 (2015). doi:10. $3390 / \mathrm{ma} 8125463$
30. F. Warmuth, M. Wormser, C. Körner, Sci. Rep. 7, 3843 (2017). doi:10.1038/s41598-017-04235-1

31. C. Körner, Y. Liebold-Ribeiro, Smart Mater. Struct. 24(2), 025013 (2015). doi:10.1088/0964-1726/24/2/025013

32. C. Körner, Int. Mater. Rev. 61(5), 361 (2016). doi:10.1080/ 09506608.2016.1176289

33. M. Suard, P. Lhuissier, R. Dendievel, J.J. Blandin, F. Vignat, F. Villeneuve, Powder Metall. 57(3), 190 (2014). doi:10.1179/ 1743290114Y.0000000093 